\title{
Non mettiamo troppa carne al fuoco: dai vegetariani ai flexitariani
}

\author{
Barbara Patella
}

PUBBLICATO: 17 MAGGIO 2019

\section{Quesito:}

Numerosi lettori ci domandano quali siano le forme più convenienti fra veganismo e veganesimo, fra vegetarianismo e vegetarismo; e, più in generale, molti altri sollevano dubbi su parole e significati riguardanti diete e abitudini alimentari.

\section{Non mettiamo troppa carne al fuoco: dai vegetariani ai flexitariani}

E a partire dal XIX secolo che diete, regimi e abitudini alimentari, dettati dai motivi più disparati (etici, igienico-salutistici, religiosi, ecologici, socioeconomici, pacifisti, antispecisti o, semplicemente, di gusto), hanno generato e continuano a generare lunghe catene lessicali, fra sinonimi, varianti e forestierismi (pescetariano, flexitariano, semivegetariano, melarismo ecc.). Cerchiamo pertanto di mettere ordine in un viluppo di parole particolarmente intricato.

Nell'ambito della scienza della nutrizione (nutrizionistica), la scelta alimentare di coloro che escludono categoricamente carne e pesce dalle proprie tavole è racchiusa in ununica parola: vegetarianismo. Secondo la letteratura scientifica, il sistema vegetariano comprenderebbe al suo interno una serie di diete, tutte accomunate dall'esclusione di prodotti carnei e ittici, ma ciascuna con una selezione personalizzata di alimenti; ne deriva una pluralità di termini tanto numerosa quanto diversificata in base ai cibi previsti. Di seguito proponiamo una tassonomia riassuntiva (a fronte dei diversi tentativi di classificazione indicati dalle principali organizzazioni scientifiche, quali la "Società Scientifica di Nutrizione Vegetariana", la "Società Italiana di Nutrizione Umana" o la "Academy of Nutrition and dietetics"):

- latto-ovo-vegetarismo (LOV): alimentazione che consente il consumo di prodotti derivati da animali vivi, quali latte, latticini, uova, miele;

- latto-vegetarismo (LV): dieta che ammette latte, latticini e miele, ma esclude le uova;

- ovo-vegetarismo (OV): dieta che elimina latte e prodotti caseari;

- veganismo (VEG): tipo di alimentazione che prevede il consumo di cibi unicamente vegetali (frutta, verdura, cereali, semi, legumi), con esclusione di qualunque prodotto di derivazione animale;

- fruttarismo: modello alimentare basato sul consumo esclusivo di frutta.

Da qualche decennio, tuttavia, rispetto alla terminologia scientifica in uso fra gli esperti di alimentazione e di nutrizione, il linguaggio comune non adopera più vegetarianismo come vocabolo iperonimo, ossia come contenitore diveganismo efruttarismo, ma usa distinguere le tre macrocategorie: da un lato, si serve di vegetarianismo per riferirsi esclusivamente al sistema dietetico che, pur eliminando la carne, prevede un consumo abituale di alimenti di derivazione animale; dall'altro, di veganismo e fruttarismo per indicare i modelli dietetici che escludono qualsiasi prodotto di provenienza animale. Il restringimento semantico è presto spiegato: col tempo i regimi alimentari che rinunciano completamente a latte, latticini, uova e miele hanno riscosso sempre più successo, fino a 
conquistare piena autonomia concettuale e, di conseguenza, nomi del tutto indipendenti.

In realtà, il flusso di parole legato al mondo dell'alimentazione ha una portata ben più elevata rispetto a quella emersa finora; ci interessa, allora, esplorare tali nomi e ripercorrerne la storia dei significati.

\section{Vegetariano, vegetarianismo, vegetarianesimo, vegetarismo, vegetarianamente, veg, veggie, vegetarista}

Iniziamo proprio da vegetariano: in qualità di aggettivo o di sostantivo, il vocabolo designa 'che (o chi) si nutre di soli cibi vegetali e non fa uso di carne, ammettendo tutt'al più l'uso di alimenti di derivazione animale, quali le uova e il latte' (Zingarelli 2019). Con tale accezione, stando al GDLI e al DELI, il termine sarebbe comparso per la prima volta nel I860 in un'opera del medico e antropologo Paolo Mantegazza, intitolata "Sulla America Meridionale. Lettere mediche". Vi è poi un'altra duplice accezione di vegetariano che, usato come aggettivo (es. ricette vegetariane), può significare 'a base di vegetali, ed eventualmente di latte, latticini, uova, con esclusione delle carni' (Garzanti 2017) oppure 'riservato ai vegetariani' (GRADIT), come nel caso di ristoranti, eventi, alberghi vegetariani.

Quanto al nome astratto che indica questo tipo di alimentazione, conosciamo tre varianti del tutto equipollenti: vegetarianismo, vegetarismo e vegetarianesimo. Quest'ultima forma, che si è diffusa molto più tardi (ultimo trentennio del Novecento) rispetto alle prime due (ultimo trentennio dell'Ottocento), si trova lemmatizzata solo nel GRADIT; in più edizioni del dizionario Garzanti, invece, vegetarianesimo si rintraccia curiosamente nella definizione di carnivorismo, ma non è posto a lemma; i numeri del web, infine, ci dicono che la forma in-esimo (I25.0oo occorrenze)risulta più frequente di quelle in -ismo (98.500 risultati per vegetarianismo e 75.300 per vegetarismo).

Alla stessa famiglia lessicale appartengono l'avverbio vegetarianamente (registrato dal GRADIT e dal Garzanti 2017) e l'accorciativo di matrice inglese veg ('vegetariano'), ugualmente attestato dai maggiori vocabolari. Un altro anglicismo in circolazione, ma finora presente solo nei dizionari bilingui ingleseitaliano, è veggie (o veggy), termine "informale" o "colloquiale" per 'vegetariano'; in verità, veggie viene adottato in italiano sia col significato di 'vegetariano' sia con quello di 'vegano', se non addirittura come unica parola per comprendere entrambe le categorie.

Aggiungiamo alla lista vegetarista, una forma non registrata nei vocabolari, ma rintracciabile nell'uso, specialmente nei contesti in cui si intende sottolineare, tramite il valore del suffisso -ista, il carattere tendenziale della scelta alimentare, alla stregua di animalista, ambientalista, idealista, riformista. Ecco un esempio:

Di conseguenza la pressione degli animalisti è rivolta verso soluzioni di tipo vegetarista o quantomeno verso un minor consumo di carne e di proteine animali [...] (Silvana Castignone, Enciclopedia delle scienze sociali, 1993, s.v. Diritti degli animali dell'Enciclopedia Treccani online).

Per quel che concerne l'etimologia, è affermata la derivazione di vegetariano dall'inglese vegetarian esito dell'unione divegetable ('verdura, ortaggio' o 'vegetale') col suffisso -arian - e quella di vegetarianismo dall'inglese vegetarianism; oltre all'origine inglese, non va peraltro trascurato l'influsso che le forme francesi végétarien e végétarisme hanno esercitato sulla nostra lingua, come segnalano il GRADIT e il DELI.

\section{E chi è vegetariano "a metà"?}

L'inesauribile produttività della lingua ha generato ben quattro espressioni per designare 'chi o che segue un'alimentazione prevalentemente ma non esclusivamente vegetariana, quindi con consumo occasionale di carne, pesce e proteine animali': 
- semivegetariano (registrato dal GRADIT e dal GDLI)

- flexitariano o flexitarian (attestato dal Garzanti 2017 e presente fra i Neologismi 2012 della Treccani)

- vegetariano flessibile (calco della voce inglese flexitarian, non entrato in lessicografia)

- reducetariano (ad oggi solo fra i Neologismi 2015 Treccani)

In questo gruppo menzioniamo un altro vocabolo ancora, accolto da più dizionari (GRADIT, GDLI, Devoto-Oli 20r8, Vocabolario Treccani online) e riservato a una tipologia alimentare più specifica: parliamo dei pescetariani, cioè di 'chi esclude dalla propria alimentazione i cibi di origine animale, ad eccezione del pesce e dei frutti di mare' (Devoto-Oli 20r8); non rara, fra l'altro, la forma pescovegetariano, che, benché non accolta in lessicografia, mostra una discreta penetrazione nella letteratura scientifica nonché nella sitografia specifica (una ricerca sul web restituisce i2.239 occorrenze della parola).

\section{Pitagorico e pitagorismo}

Prima di vegetariano e vegetarianismo, che sono coni lessicali ottocenteschi, quali nomi rinviavano ai medesimi significati? Se è vero, infatti, che vegetariano è un vocabolo "giovane", è anche vero che i vegetariani esistono da sempre.

Sin dall'epoca classica, Pitagora è considerato il caposcuola del vegetarianismo, giacché la tradizione letteraria greco-latina - si pensi in primis a Ovidio, Plutarco e Porfirio - credeva che la diffusione del modello alimentare si dovesse proprio al filosofo greco e alla teoria della metempsicosi professata dai pitagorici, cioè alla possibile reincarnazione degli uomini negli animali. Successivamente, gli scienziati del XVII e del XVIII secolo, recuperando l'immagine del Pitagora vegetariano, adottarono i termini pitagorismo e pitagorico per denotare propriamente la scelta di un'alimentazione non carnea; quest'uso si protrasse fino all'Ottocento, come spiega Carlo Anfosso in un articolo del r880:

Chi avrebbe pensato, dopo tanti secoli, di dover assistere ad una specie di rinascimento della filosofia
italica, e non più nella Magna Grecia, fatta per opera di Dio regno d'Italia, ma nella lontana Inghilterra?
Laggiù infatti rinasce in parte l'idea pitagorica colla setta dei Vegetalisti. La nuova setta non crede più
nell'Anima Universale, s'intende; non discute più di metafisica nè del sistema dell'universo; non vuole
che si parli di metempsicosi: il rinascimento pitagorico riguarda solamente il regime imposto dal
filosofo ai suoi seguaci. [...] I pitagorici si attenevano perciò ad un regime esclusivamente vegetale [...]
(Carlo Anfosso, Novità scientifiche. Pitagora in Inghilterra,"Corriere della Sera", 23/8/r88o).

Sebbene non vi sia traccia di tale accezione nella moderna lessicografia se non in senso letterario, estensivo o ironico, il significato specifico relativo all'abitudine alimentare, oggi riconosciuto sotto il nome di vegetarismo, è riportato nei dizionari storici (GDLI e Tommaseo-Bellini) sotto le voci pitagoricamente, pitagorico e pitagorismo; difatti, il GDLI definisce il vitto pitagorico quale

dieta alimentare introdotta nella scuola di Pitagora e caratterizzata principalmente dall'astensione dalle carni (assoluta o, secondo alcuni, limitata a certi animali: ciò pare in rapporto con la credenza professata dai seguaci di Pitagora nella metempsicosi, che li induceva ad astenersi da ogni forma di violenza nei confronti degli animali, in cui poteva forse nascondersi l'anima di un parente o di un amico).

Cosi pure il Dizionario d'igiene per le famiglie di Paolo Mantegazza e Neera, pubblicato a Milano nel I88I, registra la voce pitagorico (pp. 256-257) con la medesima accezione: "chiamasi con questo nome un regime composto unicamente da alimenti vegetali. [...] Ventiquattro secoli dopo Pitagora il tedesco Baltzer predicò il regime vegetale sotto il nome di vegetarianismo [...]". 


\section{Passaparola: da vegetariano a vegetaliano, da vegetalismo a veganismo}

Il vegetarianismo nacque nel I847 in Inghilterra, nel Kent, con la fondazione della "Vegetarian Society", e presto il movimento giunse anche in America, in Germania, in Francia e, solo più tardi, in Italia - la prima associazione vegetariana italiana (AVI) (cfr. http://www.vegetariani.it/) fu fondata soltanto nel I952. Cosi, a partire dalla seconda metà dell'Ottocento, si cominciò a parlare della nuova moda alimentare su numerosi giornali, tutti depositari di testimonianze linguisticamente preziose, dal momento che in nessuno di essi si perdeva occasione per intavolare la questione terminologica: dai quotidiani, come il "Corriere della Sera" e "La Stampa", alle riviste di letteratura, di chimica o di economia, quali "La Giovane Roma", "LItalia agricola" e "L'Orosi". Ad esempio, in una rivista scientifica del I893 leggiamo:

Si dovrebbe dire forse vegetaliani, ma generalmente coloro che sono per l'astensione assoluta d'ogni specie di carne permettono l'uso del latte e degli uovi ed essi si tengono d'essere vegetariani (Niccolò de' Colli, Vegetarianismo e Vegetariani, "L'Orosi. Giornale di chimica, farmacia e scienze affini", Firenze, anno XVI, n.2, febbraio I893, p. 52).

L'articolo citato corrisponde alla traduzione di uno scritto del dottor Menard uscito sulla rivista francese "Cosmos", e proprio le numerose traduzioni da testi francesi, tedeschi, inglesi, russi hanno contribuito a una proliferazione di forme e a un'oscillazione di significati.

Sul problema terminologico scrisse anche Costantino Arlia, il magistrato, filologo e lessicografo ricordato soprattutto per il Lessico dellinfima corrotta italianità (I877). Nel i899 il "puntiglioso linguista" - cosi lo definì Carducci - pubblicò un articolo sul settimanale "I diritti della scuola", nella sezione "Noterelle di lingua", in cui lamentava la popolarità delle voci vegetarismo, vegetarista e vegetariano, a parer suo errate rispetto alle forme vegetalismo, vegetalista (quest'ultima già incontrata nel brano di Anfosso)e vegetaliano: secondo Arlia la seconda serie lessicale, derivando da vegetale, rispecchiava più fedelmente la scelta di una dieta a base di alimenti vegetali, a differenza delle forme con $r$, riconducibili erroneamente a vegetare 'vivere e crescere, detto delle piante' (Zingarelli 2org), etimo inesatto (cfr. C. Arlia, Vegetarismo, Vegetarista, "I diritti della scuola", Supplemento letterario dell'II/II/I899, n. 6, pp. 2I-22).

Si contano numerose altre testimonianze, quale, ad esempio, quella di un articolo del "Corriere della Sera":

Quanti vegetalisti... (Tutti dicono «vegetariani» perché la dolce mania è venuta di fuori, come - o sorridente Italia! - tutte le manie, e con la mania il nome; ma, insomma, i signori «vegetariani» vogliono nutrirsi di vegetali o vogliono, Dio li protegga, arrivare alla imitazione della pianta e contentarsi, come sembra indicar la parola, di «vegetare»? Diciamo vegetalisti, dunque: neologismo per neologismo, bisogna scegliere il meno disadatto al genio della lingua) (Il violino complice della bistecca, "Corriere della Sera", I6/II/1922).

Dunque, si diceva vegetariano o vegetaliano? Vegetalismo o vegetarismo? Vegetalista o vegetarista? Inizialmente i termini erano adoperati un po' promiscuamente e le forme citate indicavano indistintamente qualcuno che eliminasse la carne dalla propria alimentazione, senza distinguere chi rifiutasse anche latte, latticini, uova e miele e chi no. Insomma, era ancora in fase di assestamento la terminologia e lontana la divisione che conosciamo oggi, secondo cui vegetalista e vegetaliano sono sinonimi di vegano e non di vegetariano; le prime testimonianze della attuale distinzione risalgono al I927 per vegetaliano e al I93I per vegetalista: 
I vegetaliani di Luynes non han niente a che fare coi vegetariani. Il burro, il latte, e le uova li separano. I vegetaliani respingono con orrore questi alimenti che, se non sono propriamente carnei, con la carne delle mucche e delle galline hanno avuto, in origine, contatti strettissimi (I vegetaliani, "Corriere della Sera", 23/4/1927).

Distinguiamo innanzi tutto i vegetariani dai vegetalisti: i primi accettano tutti gli alimenti vegetali e animali e fanno una sola eccezione per la carne; i secondi invece respingono addirittura tutti gli alimenti di origine animale e quindi anche il latte, le uova, il burro, il formaggio, il lardo (Carne o verdura?, "Minerva. Rivista delle riviste", vol. LI, n. 5, I5/3/1931, p. I87).

Come si è potuto vedere, quindi, prima di vegan, vegano, veganista e veganiano (voci censite dalla lessicografia fin dagli anni Novanta) erano vegetaliano e vegetalista i termini deputati a indicare, con funzione sostantivale, 'chi segue una dieta completamente priva di prodotti direttamente o indirettamente animali' o, con funzione aggettivale, 'che è a base di alimenti di origine vegetale'.

Non sorprende che in breve tempo vegano abbia imperiosamente scavalcato vegetaliano (ormai destinato a tramontare): vegetaliano e vegetariano veicolavano significati diversi attraverso parole troppo simili, ragion per cui occorreva attuare una distinzione lessicale più efficace. Stesso discorso per vegetalismo, soppiantato da veganismo (o veganesimo, variante accolta solo nel Garzanti 2017 e tendenzialmente più frequente nella stampa, per esempio sulla "Repubblica" o sul "Corriere"); per giunta, in luogo diveganismo, è possibile trovare le forme analitiche vegetarianismo stretto, vegetarianismo puro o vegetarianismo integrale, seppur sporadicamente.

Ma qual è l'origine di vegano? Vegano è un adattamento italiano della voce inglese vegan (forma contratta di vegetarian, coniata nel I944 da Donald Watson, fondatore della Vegan Society) ed è entrato nella nostra lingua negli anni '8o del secolo scorso; per di più, dai primissimi anni Duemila, si è affermata anche la forma abbreviata veg (omonima, peraltro, dell'accorciativo veg derivante da vegetariano).

Infine, bisogna precisare un ulteriore aspetto: la differenza tra vegetariani e vegani non interessa soltanto l'alimentazione, bensi l'intero stile di vita; a tal proposito alcuni dizionari definiscono il veganismo quale 'movimento e stile di vita che esclude l'uso di tutti i prodotti di origine animale, anche per l'abbigliamento, e che in generale rifiuta ogni forma di sfruttamento degli animali, dalla tosatura alle corride' (Garzanti 20I7).

Chiudiamo la lunga serie lessicale con un sottotipo della dieta vegana basato sul consumo di cibi vegetali non cotti, il cosiddetto crudismo vegano ( o crudiveganismo), che conosce oltre I3.ooo occorrenze sul web, e un migliaio di risultati su Google Libri, mentre risulta sconosciuto ai dizionari.

\section{Fruttarismo, fruttarianismo, fruttarianesimo}

Menzioniamo, in ultimo, la terza macrocategoria di cui si è fatto cenno all'inizio: una "persona che limita la propria alimentazione a frutta fresca, biologica e matura' (Devoto-Oli 20I8) può essere definita fruttariano o fruttarista e la 'adozione di una dieta composta esclusivamente da frutta' (GRADIT) va sotto il nome di fruttarianismo, fruttarianesimo, fruttarismo (il Garzanti 2017 è l'unico a porre a lemma tutte e tre le varianti). Di questa dieta esiste una forma ridotta all'osso, o meglio, ridotta al consumo di sole mele, il melarismo, e coloro che lo praticano sono noti col nome di melariani. Né melarismo né melariano sono censiti dai dizionari, ma negli ultimi anni sono divenuti termini piuttosto popolari fra web, tv, radio e libri. 
In conclusione, di fronte a una gamma di parole e di forme tanto vasta, risulta evidente la forza esercitata dall'analogia linguistica nel promuovere formazioni corradicali sempre nuove, sia nello scritto sia nel parlato. E dal serio al faceto è un attimo: si passa dai sassariani creati da Lercio ai sedanisti e ai briciolisti di Crozza fino ad arrivare al respirianesimo che, fra verità e bufale, ha fatto tanto discutere e, a quanto pare, non si tratterebbe di una fake news, bensi di una filosofia di vita realmente praticata e meglio conosciuta come alimentazione pranica.

\section{Cita come:}

Barbara Patella, Non mettiamo troppa carne al fuoco: daivegetariani ai flexitariani , "Italiano digitale", 2019, IX, 2019/2 (aprile-giugno)

DOI: $10.35948 / 2532-9006 / 2020.3115$

Copyright 2019 Accademia della Crusca

Pubblicato con licenza creative commons CC BY-NC-ND 